\title{
Difficulties of CIDP Diagnosis
}

\author{
Małgorzata Lukawska, Anna Potulska-Chromik* and Anna Kostera-Pruszczyk \\ Department of Neurology, Medical University of Warsaw, Poland, ERN EURO-NMD \\ *Corresponding author: Anna Potulska-Chromik, Department of Neurology, Medical University of Warsaw, Poland. \\ To Cite This Article: Małgorzata Łukawska, Anna Potulska-Chromik, Anna Kostera-Pruszczyk, Difficulties of CIDP Diagnosis. Am J Biomed Sci \\ \& Res. 2021 - 11(4). AJBSR.MS.ID.001653. DOI: 10.34297/AJBSR.2021.11.001653.
}

Received: 制 December 12, 2020; Published: 眥 January 15, 2021

\begin{abstract}
Abbreviations: CIDP: Chronic Inflammatory Demyelinating Polyneuropathy; NCS: Nerve Conduction Study; CSF: Cerebrospinal Fluid; GBS: GuillainBarré syndrome; HNPP: Hereditary Neuropathy with Pressure Palsies; MMN: Multifocal Motor Neuropathy; IVIg: Intravenous Immunoglobulin; DADS: Distal Acquired Demyelinating Symmetric Neuropathy; MADSAM: Multifocal Acquired Demyelinating Sensory and Motor Neuropathy; INCAT: Inflammatory Neuropathy Cause and Treatment
\end{abstract}

\section{Introduction}

Chronic inflammatory demyelinating polyneuropathy (CIDP) is an autoimmune neuropathy characterized by weakness and/or sensory symptoms developing over more than 8 weeks and hipo or areflexia. It is worth emphasizing that CIDP is the most common treatable neuropathy in the world [1]. It is important to include it in the differential diagnosis, as some studies suggest that CIDP is misdiagnosed in up to $47 \%$ of patients [2]. The reason of this phenomen is multifactorial, from diverse clinical presentation, especially misleading in atypical variants, through electrodiagnostic pitfalls to objective estimation of the treatment response. In this article we discuss the difficulties of diagnosis CIDP with particular emphasis on atypical variants.

\section{CIDP Epidemiology and Diagnostic Criteria}

The prevalence of CIDP is $0.46-1.83$ persons per 100.000 $[3,4]$. The disease occurs in all age groups, but the incidence increases with age, peaking in middle-aged people and is slightly more common in men [4]. In most cases symptoms progress over 2 months, however, in about $18 \%$ of patients the development of symptoms can be acute, mimicking Guillain-Barre syndrome (less than 4 weeks) or subacute (4-8 weeks) [5]. CIDP as an autoimmune disease can be preceded by an infection, mostly respiratory tract infection or gastroenteritis [6].

The typical clinical presentation is progressive, recurrent or stepwise symmetrical proximal and distal weakness with sensory symptoms in all extremities, in some cases additional cranial nerves involvement can also occur [7]. The course of the disease in younger patients is often relapsing-remitting, whereas in the elderly it is chronic progressive [8]. Nerve conduction study (NCS) demonstrates demyelinating changes: slowing of conduction velocity, prolongation of distal latency, prolongation or absence of F-wave, partial motor conduction block, abnormal temporal dispersion and prolongation of distal CMAP duration [7]. Supportive criteria include the cytoalbuminologic dissociation in the cerebrospinal fluid (CSF) elevated protein level without pleocytosis (lecocyte count $<10 / \mathrm{mm} 3$ ), gadolinium enhancement and/or hypertrophy of nerve roots or plexuses in imaging studies (MRI or ultrasound). Objective improvement to immunomodulatory treatment is also an important clinical feature of CIDP [7].

The sural biopsy is no longer recommended in a routine diagnosis. Differential diagnosis includes Guillain-Barré syndrome (GBS), multifocal motor neuropathy (MMN), hereditary neuropathy with pressure palsies (HNPP), hereditary sensorimotor polyneuropathies, especially Charcot-Marie Tooth disease (CMT), neuropathy associated with diabetes mellitus or vasculitis [5]. Based on the clinical and electrophysiological criteria CIDP diagnosis can be definite, possible or probable [7]. The first-line treatment are intravenous immunoglobulin (IVIg) or corticosteroids [7]. If the response to treatment is absent or incomplete the other therapy is started, in the next step immunosuppressive or immunomodulatory 
drugs are added [7]. The prognosis is rather good, however, most of the patients require long term treatment [7].

\section{Atypical Variants of CIDP - Clinical Criteria Pitfalls}

As mentioned in the Background an atypical presentation can be misleading and cause delay in the diagnosis. Initially $31-48 \%$ of patients present with an atypical CIDP, but over time most of them develops the typical clinical features, leaving only $18 \%$ patients with an atypical CIDP variant in the long-term follow-up [9]. The criteria for atypical CIDP has changed throughout the years [10]. Current EFNS/PNS criteria [7] distinguish the following atypical variants:

\section{Predominantly dista}

Distal acquired demyelinating symmetric neuropathy (DADS)

\section{Asymmetric}

Multifocal acquired demyelinating sensory and motor neuropathy (MADSAM, Lewis-Sumner syndrome)

3. Focal

4. Pure motor

5. Pure sensory
6. Chronic immune sensory polyradiculopathy

\section{Distal Acquired Demyelinating Symmetric Neuropathy (DADS)}

DADS is characterized by distal involvement of motor and sensory symptoms in the lower limbs, later symptoms may occur in the upper limbs. Exclusion criteria include proximal distribution of symptoms, pure motor variants, initial symptoms in the upper limbs and cranial nerves involvement [10].

\section{Multifocal Acquired Demyelinating Sensory and Motor Neuropathy (MADSAM; Lewis-Sumner Syndrome)}

In the MADSAM symptoms are multifocal, asymmetric, sensory loss is distal, while weakness usually affects upper more often than lower limbs, and is asymmetric [7]. Cranial nerves palsy has been described in MADSAM. Ataxia, cramps or autonomic dysfunction cam also coexist [10].

\section{Focal CIDP}

Focal CIDP affects motor and sensory fibers of one limbs' nerves, in the plexus or more peripherally [7]. In Table 1 there are summarized most characteristic information regarding atypical variants of CIDP and the most common mimics [5].

Table 1: Atypical variants of CIDP.

\begin{tabular}{|c|c|c|c|}
\hline Atypical Variant of CIDT & Frequency & Red Flags & Main Differential Diagnosis \\
\hline DADS & $2-10 \%$ & $\begin{array}{c}\text { Symptoms are distally, firstly in the lower limbs, in the } \\
\text { upper limbs after }>1 \text { year }\end{array}$ & $\begin{array}{c}\text { MMN, HNPP, Vasulitis, Neuralgic, } \\
\text { Amyotrophy, SHA } \\
\text { Diabetes Mellitus }\end{array}$ \\
\hline MADSAM & $8-15 \%$ & $\begin{array}{c}\text { Multifocal motorsensory distribution, } \\
\text { NCS: Persistent or probable CB }\end{array}$ & $\begin{array}{c}\text { MMN, HNPP, Vasulitis, Neuralgic, } \\
\text { Amyotrophy, SHA }\end{array}$ \\
\hline Focal & $1 \%$ & Sensorimotor Symptoms in one limb & $\begin{array}{c}\text { Araneoplastic Neuopathies, Connective Tissue } \\
\text { Disease }\end{array}$ \\
\hline Pure Sensory & $4-35 \%$ & No motor involvement, \\
CISP & $4-35 \%$ & $\begin{array}{c}\text { No automatic dysfunction } \\
\text { At least } 2 \text { of 3 Abnormal SSEP, Contrast enhancement } \\
\text { and or hypertrophy of nerve roots or plexus, Cytoal- } \\
\text { buminologic dissociation in CSF }\end{array}$ & $\begin{array}{c}\text { Paraneoplastic Neuopathies, Connective Tissue } \\
\text { Disease }\end{array}$ \\
\hline Pure Motor & $4-10 \%$ & Only motor symptoms, No autonomic dysfunction & Guillain Barre Syndrome, Motor Neuron \\
\hline
\end{tabular}

Note: CIDP: Chronic Inflammatory Demyelinating Polyradiculoneuropathy; DADS: Distal Acquired Demyelinating Symmetric Neuropathy; MADSAM: Multifocal Acquired Demyelinating Sensory And Motor Neuropathy; NCV: Nerve Conduction Study; CB: Conduction Block; MMN: Multifocal Motor Neuropathy; HNPP-Hereditary Neuropathy with Liability to Pressure Palsies; IgM-Immunoglobulin M; MGUS: Monoclonal Gammopathy of Undetermined Significance; MAG: Myelin-Associated Glycoprotein; SMA: Spinal Muscular Atrophy; CISP: Chronic Immune Sensory Polyradiculopathy; SSEP: Somatosensory Evoked Potential; CSF: Cerebrospinal Fluid.

\section{Pure Sensory}

The exclusion criteria in this variant are any motor involvement, both clinical and electrophysiological, and autonomic dysfunction. Sensory dysfunction may present not only as paresthesia but also as ataxia and may localize in the face area [10].

\section{Chronic Immune Sensory Polyradiculopathy (CISP)}

CISP is characterized by clinical pure sensory involvement with no changes in NCS neither in motor fibres nor sensory but with at least two out of three abnormalities in additional studies (magnetic resonance imaging, somatosensory evoked potential, cytoalbuminologic dissociation in CSF) [10]. 


\section{Pure Motor}

This variant includes only motor symptoms and may involve cranial nerves but sensory disturbances or dysautonomia are never present [10].

\section{Electrodiagnostic and Supplementary Criteria Pitfalls}

One of the most important steps to an accurate diagnosis of CIDP is examining a sufficient number of peripheral nerves according to the EFNS recommendations at least 4 nerves (median, ulnar, peroneal and tibial) or more, if the diagnostic criteria are not met [7]. Study by Rajabelly et al. [11] has proved that assessing 5-8 nerves is associated with almost $100 \%$ diagnostic sensitivity. Still, in cases where only the proximal nerves' segments, within the plexus or spinal roots are involved, these changes may not be confirmed in a routine NCS [9]. Other tests such as the CSF parameters can aid the diagnosis, but eg. protein concentration in CSF may be influenced by factors such as age, comorbid conditions including spinal stenosis or diabetes mellitus [9]. In the patients with diabetes mellitus a score created with the combination of clinical and laboratory features has been developed to aid CIDP diagnosis [12].

The response to immunomodulating treatment should be objectively confirmed with appropriate scales e.g. INCAT (Inflammatory Neuropathy Cause and Treatment (INCAT) Disability Score), functional tests such as Medical Research Council scale for measuring muscle strength, grip strength testing, 6 minute walking tests [9]. Relying only on the patient's impression may often be misleading [9].

\section{Summary}

To sum up the typical CIDP should not cause diagnostic difficulties with the appropriate work-up. Diagnosis of atypical variants may be misleading. It is important to remember about the diverse clinical and electrophysiological presentation as well as results from ancillary tests. The incorrect diagnosis is associated with the lack of treatment or the unnecessary, costly and potentially harmful therapy.

\section{References}

1. Mathey EK, Park SB, Hughes RA, John D Pollard, Patricia J Armati, et al. (2015) Chronic inflammatory demy-elinating polyradiculoneuropathy: from pathology to phenotype. J Neurol Neurosurg Psychiatry 86(9): 973-985.

2. Allen JA, Lewis RA (2015) CIDP diagnostic pitfalls and perception of treatment benefit. Neurology 85(6): 498-504.

3. Lunn MP, Manji H, Choudhary PP, PP Choudhary, RA Hughes, et al. (2008) Chronic inflammatory demyelinating polyneuropthy a prevalence study in south east England. J Neurol Neurosurg Psychiatry 66(5): 677-680.

4. Iijima M, Koike H, Hattori N, A Tamakoshi, M Katsuno, et al. (2009) Prevalence and incidence rates of chronic inflammatory demyelinating polyneuropathy in the Japanese population. J Neurol Neurosurg Psychiatry 79(9): 1040-1043.

5. Bunschoten C, Jacobs BC, Van den Bergh P, David R Cornblath, Pieter A van Doorn, et al. (2019) Progress in diagnosis and treatment of chronic inflammatory demyelinating polyradiculoneuropathy. Lancet Neurol 18(8): 784-794

6. Peltier AC, Donofrio PD (2012) Chronic inflammatory demyelinating polyradiculoneuropathy: from bench to bedside. Semin Neurol 32(3):187-195.

7. PYK Van den Bergh, RDM Hadden, P Bouche, DR Cornblath, A Hahn (2010) European Federation of Neurological Societies/Peripheral Nerve Society Guideline on management of paraproteinemic demyelinating neuropathies: Report of a Joint Task Force of the European Federation of Neurological Societies and the Peripheral Nerve Society-first revision. J Peripher Nerv Syst 17(3):356-363.

8. Hattori N, Misu K, Koike H, et al. (2001) Age of onset influences clinical features of chronic inflammatory demyelinating polyneuropathy. J Neurol Sci 184(1): 57-63.

9. Allen JA (2020) The Misdiagnosis of CIDP: A Review. Neurol Ther 9(1): 43-54.

10. Doneddu PE, Cocito D, Manganelli F, Raffaella Fazio, Chiara Briani, et al (2019) Atypical CIDP: diagnostic criteria, progression and treatment response. Data from the Italian CIDP Database. J Neurol Neurosurg Psychiatry 90(2): 125-132.

11. Rajabally YA, Adams D, Latour P, Shahram Attarian (2016) Hereditary and inflammatory neuropathies: a review of reported associations, mimics and misdiagnoses. J Neurol Neurosurg Psychiatry 87(10): 10511060

12. Lotan I, Hellman MA, Steiner I (2015) Diagnostic criteria of chronic inflammatory demyelinating polyneuropathy in diabetes mellitus. Acta Neurol Scand 132(4): 278-283. 